\title{
Frontières
}

\section{La mort dans les camps de concentration}

\section{Luba Jurgenson}

Volume 19, numéro 1, automne 2006

Enjeux politiques et mort

URI : https://id.erudit.org/iderudit/016632ar

DOI : https://doi.org/10.7202/016632ar

Aller au sommaire du numéro

Éditeur(s)

Université du Québec à Montréal

ISSN

1180-3479 (imprimé)

1916-0976 (numérique)

Découvrir la revue

Citer cet article

Jurgenson, L. (2006). La mort dans les camps de concentration. Frontières, 19(1), 25-30. https://doi.org/10.7202/016632ar

\section{Résumé de l'article}

Après avoir analysé les processus de mise à mort et de destruction (ou enterrement anonyme) des corps dans les camps nazis et soviétiques, on examinera les rites élaborés au sein de la société concentrationnaire autour des mourants et des cadavres ainsi que l'état physique et psychologique particulier entre la vie et la mort qui fait l'objet de constructions narratives complexes dans les récits des survivants. On interrogera les stratégies visant à représenter ces états-limite et à reconstituer les espaces conçus pour produire du néant. On tentera de montrer que le texte du témoignage, au-delà de son objectif explicite - transmettre l'expérience - est un texte agissant investi de fonction de sépulture.
Ce document est protégé par la loi sur le droit d'auteur. L'utilisation des services d'Érudit (y compris la reproduction) est assujettie à sa politique d'utilisation que vous pouvez consulter en ligne.

https://apropos.erudit.org/fr/usagers/politique-dutilisation/ 


\section{Résumé}

Après avoir analysé les processus de mise à mort et de destruction (ou enterrement anonyme) des corps dans les camps nazis et soviétiques, on examinera les rites élaborés au sein de la société concentrationnaire autour des mourants et des cadavres ainsi que l'état physique et psychologique particulier entre la vie et la mort qui fait l'objet de constructions narratives complexes dans les récits des survivants. On interrogera les stratégies visant à représenter ces états-limite et à reconstituer les espaces conçus pour produire du néant. On tentera de montrer que le texte du témoignage, au-delà de son objectif explicite - transmettre l'expérience - est un texte agissant investi de fonction de sépulture.

Mots clés: confiscation - limite représentation - texte-sépulture trace - transmission.

\section{Abstract}

After analyzing the processes of killing and destroying (anonymous burial) of bodies in Nazi and Soviet camps, this paper examines the rites created in the concentration camp communities around the dying, the cadavers, and the particular physical and psychological states between life and death that become the object of complex narrative constructions in the stories of survivors. We will investigate the strategies used to represent these limit-states and those used to reconstitute spaces designed to produce a void. We will attempt to show that the witness story, beyond its explicit objective - recounting an experience - is a living text invested with a sepulture function.

Keywords: confiscation - limit representation - sepulture text trace - transmission.

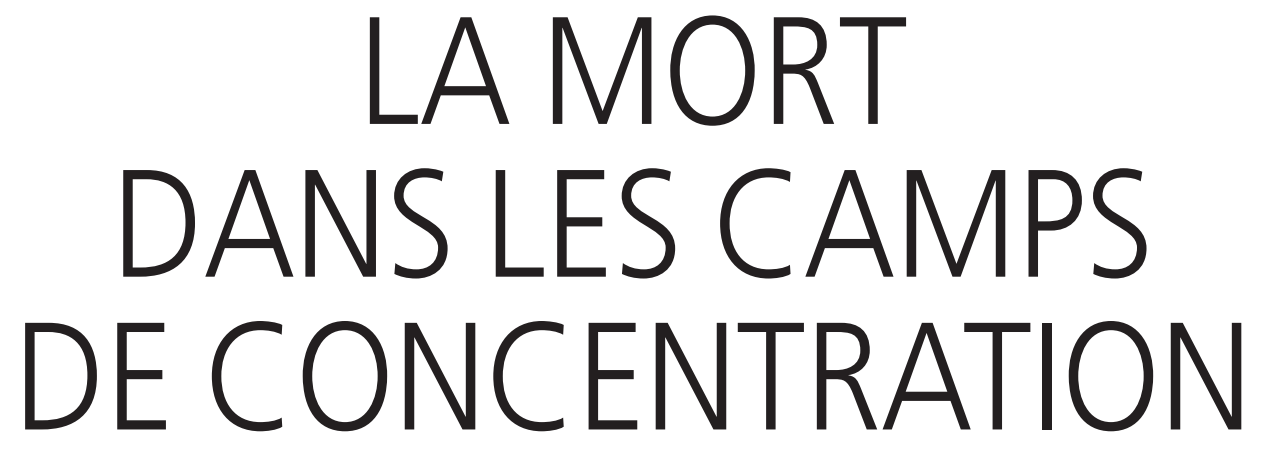

\author{
Luba Jurgenson, \\ Paris-IV Sorbonne, études slaves.
}

Dans les récits sur les camps de concentration et d'extermination, la mort apparaît comme une catégorie essentielle, une réalité quotidienne qui entoure, tout au long de leur expérience, les détenus toujours à la limite de la disparition. Elle oriente les comportements, modèle le temps et l'espace et, compagne fidèle du Häftlinge ou du zek même après sa libération, s'insinue au plus profond des mots lorsqu'il s'agit de dire les camps. Unique garante de la légitimité du témoignage ${ }^{1}$, elle continue, longtemps après, à peser sur la conscience du survivant et à invalider sa parole, pour devenir le lieu même d'où cette parole improbable peut, finalement, jaillir.

\section{LA MORT COMME FINALITÉ}

L'objectif officiel des camps de concentration soviétiques était, d'un côté, la rééducation $^{2}$, de l'autre, la production. L'un des objets produits en série par ces camps était le cadavre. Ce produit final était le résultat d'une suite de confiscations que subissait le détenu, dépouillé de ses effets personnels, de sa mémoire, de ses cheveux (pour les hommes), de son nom parfois. Au bout de la chaîne, le corps lui-même apparaissait comme une "possession» illégitime, un surplus à restituer à l'État.
Quant aux camps d'extermination nazis, leur objectif unique fut la production non pas de corps, mais de néant ${ }^{3}$. Le processus de confiscation est allé ici encore plus loin: même leur propre mort fut confisquée aux victimes. Coupables du crime d'être nées, elles devaient disparaître intégralement comme si elles n'avaient jamais existé. Leur mort n'était pour ainsi dire pas une mort, mais la réparation de l'anomalie que constituait leur existence. Là où il aurait dû y avoir du rien, il y avait quelque chose. Ce quelque chose devait retourner au rien. Sur le chemin du quelque chose au rien, il y avait un résidu, le cadavre. D'où la nécessité d'en détruire non seulement toutes les traces physiques, mais également les moindres traces mémorielles, y compris la trace du meurtre dans les représentations des victimes elles-mêmes, abusées sur leur sort jusqu'au dernier moment. Les victimes acheminées vers les centres de mise à mort et gazées immédiatement n'étaient pas enregistrées, elles disparaissaient purement et simplement.

Les Allemands se sont mis à détruire les corps, auparavant entassés dans des fosses communes, à partir de la fin de 1942. Dès l'été 1942, on a lancé la construction de plusieurs chambres à gaz dans les centres de mise à mort, afin d'accélérer le processus de destruction ${ }^{4}$. Il s'agissait de réaliser en parallèle deux objectifs urgents : tuer le plus 
de Juifs possible et effacer les traces des massacres. La technique mise en place pour assurer aux usines de la mort le rendement maximal a permis conjointement d'élaborer le dispositif d'effacement le plus efficace. Souci d'échapper à la responsabilité ou nécessité de projeter rétrospectivement les victimes dans le néant? Il serait vain de séparer ces deux objectifs, car le refus de la responsabilité juridique était en même temps négation de l'existence des victimes ${ }^{5}$.

Il en fut autrement dans les camps soviétiques. Le concept d'ennemi du peuple, tout fluctuant et arbitraire qu'il était, impliquait, dans la fiction judiciaire qui accompagnait les arrestations, une "faute» commise par l'individu au cours de sa vie consciente ${ }^{6}$. Cette faute variait de purge en purge au gré des besoins de l'État soviétique, et la chasse à l'ennemi, la "découverte » chaque fois de nouveaux foyers d'infection antisoviétique et leur «démantèlement » augmentaient la puissance de l'État. La mort était un châtiment pour un crime commis et le corps châtié, un gage de cette puissance. L'amoncellement de cadavres, dans cette optique, contribuait à l'immortalité du régime et, partant, à l'immortalité personnelle du tyran. Les autorités n'éprouvaient pas le besoin de faire disparaître intégralement le corps, il leur suffisait d'effacer l'identité du défunt. Le cadavre n'était d'ailleurs pas gênant dans la mesure où on lui retirait son nom et son histoire: il ne constituait plus de trace. Les immenses espaces de l'URSS offraient des emplacements commodes pour les charniers. Et puis, les autorités soviétiques ne craignaient pas d'être jugées pour les crimes commis. Elles ne l'ont jamais été, du reste.

Toutefois, les taux de mortalité dans les camps soviétiques étaient gardés secrets. Lorsque les familles recevaient des avis de décès, les causes de la mort, généralement la faim et l'épuisement, n'y figuraient pas du tout ou étaient falsifiées. Bien des décès ne furent révélés qu'après la chute du régime. Les peines de dix ans sans droit de correspondance étaient en vérité des peines de mort.

Dans les camps, les fusillades avaient lieu la nuit. Les coups de feu étaient couverts par le vrombissement des camions. Parfois, les condamnés n'étaient pas fusillés à l'intérieur de leur camp, mais conduits vers des prisons spécialisées, par exemple, la Serpentine, célèbre lieu d'extermination de la Kolyma, ou la «maison Vaskov», prison de Magadan.

Il existait, dans les administrations des camps soviétiques, une certaine tension entre le centre et la périphérie. La machine à démasquer et à tuer prononçait des condamnations à l'encontre des «politiques » et les affectait à des travaux physiques durs, ce qui signifiait une mort imminente. Par ailleurs, cette même machine réclamait aux chefs des camps qui recevaient ces ennemis du peuple qu'ils remplissent la norme de production, ce qui était impossible compte tenu du taux de mortalité sur les chantiers. Ces chefs, menacés de se retrouver eux-mêmes derrière les barbelés, tentaient de résoudre le problème en se retournant contre les commandants des lagpounkt particulièrement meurtriers, dont certains ont été de ce fait relevés de leurs fonctions ou fusillés ${ }^{7}$. Ces conflits locaux constituaient une chance de survie pour les détenus. Toutefois, ce processus peut s'expliquer également par la logique de la machine totalitaire elle-même, où les bourreaux sont périodiquement remplacés par d'autres bourreaux.

On dispose de deux sortes de témoignages directs sur les processus de mise à mort dans les camps nazis: ceux des membres des Sonderkommandos ${ }^{8}$ et les dépositions des nazis chargés de l'extermination ${ }^{9}$. À cela s'ajoutent les témoignages sur le Sonderkommando et ceux produits par les Alliés à propos de ce qu'ils ont découvert à l'ouverture des camps ${ }^{10}$. De nombreuses études ont été menées sur l'appareil d'extermination nazi ${ }^{11}$.

En ce qui concerne les camps soviétiques, dans la mesure où il n'existait pas d'appareil de mise à mort spécifique, tous les survivants abordent la question de la mort $^{12}$. Ils s'accordent pour dire que c'était le travail qui tuait. Organisé de façon à annihiler même les plus vigoureux en deux à trois semaines, le labeur quotidien était un outil de meurtre de masse particulièrement efficace.

Notons également que dans le système concentrationnaire nazi, il existait de nombreux camps qui n'étaient pas équipés d'appareils de destruction ainsi que d'autres qui, comme le complexe des camps d'Auschwitz, Majdanek, Mauthausen ont fonctionné à la fois comme camp de concentration et camp d'extermination ${ }^{13}$. La mort par épuisement $\mathrm{y}$ a fait de très nombreuses victimes dans toutes les catégories des détenus astreints au travail ${ }^{14}$.

\section{LES RITES}

La mort des détenus a donné lieu à de nombreux rites spécifiquement réservés aux espaces concentrationnaires, entièrement imposés par les autorités des camps. Certains d'entre eux, comme l'extraction des dents en or, étaient communs aux camps nazis et soviétiques, mais dans l'ensemble, on peut dire que chaque système a élaboré son rituel propre, dû à divers facteurs: les particularités des sites, le rapport à la nature et à la technique, le degré d'organisation, l'homogénéité des espaces, leur plus ou moins grande centralisation, le besoin de cacher les camps aux yeux du monde, enfin, l'image et le statut des victimes.

La ritualisation des espaces de la mort commençait du vivant de la victime. Les vocables qui servaient à désigner cette dernière visaient à en faire un détritus aux yeux des autres et à ses propres yeux, la retranchant d'emblée du monde humain et désignant une zone de non-existence. Les deux systèmes recouraient à des mots tels que « rats », "vermine», "contagion» et d'autres termes qui niaient l'appartenance de l'ennemi à la communauté humaine. On ne s'adressait aux détenus qu'en les insul$\operatorname{tant}^{15}$. Primo Levi raconte une scène où un jeune kapo se fait rabrouer par un milicien pour avoir dit «Mann» (hommes) à propos de détenus, la seule formule autorisée étant Häftlinge (détenu).

Dépouillé de ses vêtements et anonyme, le corps était généralement déposé dans une fosse commune dans la plupart des camps soviétiques. Il portait, attachée à la cheville ou au genou, une plaquette en bois (birka) avec le numéro du dossier pénitentiaire $^{16}$. Dans certains camps, les crânes des cadavres étaient brisés à coup de pic ou encore, on leur enfonçait une lame dans le cœur, afin d'empêcher toute tentative d'évasion. Varlam Chalamov fait également état d'une pratique inédite, liée à la difficulté de transporter les corps des évadés à la Kolyma: exécutés sur place, ces derniers n'étaient pas ramenés au camp, on se contentait de leur couper les mains afin de pouvoir les identifier d'après leurs empreintes (Chalamov, 2003, p. 1245-1280).

Il est tout à fait logique que le rite d'enterrement ait été interdit dans ces lieux où la mort était justement l'aboutissement du processus de détention ${ }^{17}$. En tant qu'objet final de l'industrie mise en place, le corps ne pouvait être l'objet symbolique, le signe. La création de la trace est incompatible avec une mise à mort sérielle. L'anéantissement massif exige précisément l'absence de toute trace: l'absence de l'absence. Le monde doit se refermer sur la victime comme les vagues de la mer ${ }^{18}$. Un corps mis en terre dans un cimetière révèle le surgissement du vide dans le monde. Caché, il rend manifeste l'absence. Dans les camps, les cadavres et l'odeur de la mort étaient partout, et pourtant, on s'acharnait à entretenir la dynamique d'un monde opaque sans aucun «blanc», aucune béance par où la mort aurait pu se faire voir et entendre comme événement.

Dans les camps nazis, il était interdit de prononcer les mots «corps» ou «morts» à propos des victimes. Ces mots étaient placés par figuren, poupées, marionnettes ou figurines ${ }^{19}$, ou encore Schmattes, chiffons. Devenu représentation et non plus 
LA RITUALISATION DES ESPACES DE LA MORT COMMENÇAIT DU VIVANT DE LA VICTIME. LES VOCABLES QUI SERVAIENT À DÉSIGNER CETTE DERNIÈRE VISAIENT À EN FAIRE UN DÉTRITUS AUX YEUX DES AUTRES ET À SES PROPRES YEUX, LA RETRANCHANT D’EMBLÉE DU MONDE HUMAIN ET DÉSIGNANT UNE ZONE DE NON-EXISTENCE.

matière, emballage vide, détritus, le corps était comme arraché à la mort, transformé en image. Or, le rapport du cadavre à l'humain n'est pas métaphorique, et c'est là probablement la source de l'effroi qu'il inspire. Le cadavre ne peut pas figurer le vivant, il est même la trace sensible, matérielle de ce qui ne peut d'aucune manière être figuré : la mort. C'est donc cette impossibilité de figurer que l'on nie en introduisant $\mathrm{du}$ «figurer» là où il ne peut $\mathrm{y}$ en avoir. Notons que si la confection d'une poupée qui imite l'humain suppose un « faire » créatif, le cadavre, ici, s'obtient par un meurtre. L'interdiction de dire «mort » vise à évacuer cette dimension et à disculper le meurtrier. Pas de destruction, donc pas de criminel ni de victime. Tout le projet d'extermination est résumé dans cette simple substitution d'une parole à une autre. Car le dire mémoriel se construit à partir de ce vide que la mort creuse dans le langage. Faire apparâ̂tre une "figurine » là où il y a passage de la vie à la mort, c'est attenter au langage dans sa fonction de témoignage. La « mort » de la mort, censée assurer une vie éternelle aux bourreaux, relègue les victimes à l'inexistence et au silence éternel.

\section{ENTRE LA VIE ET LA MORT}

Les rites d'enterrement permettent à la communauté de préserver l'illusion de son immortalité. Au camp, en revanche, la mort d'un individu n'est pas un événement. Dans le corps collectif, chaque corps particulier est déjà de trop et sa disparition n'ébranle pas. Pourtant, la destruction d'un grand nombre menace la communauté tout entière, dont l'existence n'apparaît alors plus que comme un sursis.

Chez les survivants des camps, ce qu'Elias Canetti appelle «la passion de survivre» (Canetti, 1986) ne donne pas lieu au syndrome de l'immortalité et ne procure pas de jouissance. Bien au contraire, de nombreux avantages de cette survie leur sont souvent confisqués du fait que le camp ne les quitte jamais véritablement. Plutôt que de devenir invulnérables et de sentir leur moi augmenter sa puissance comme cela peut être le cas dans les guerres et les épidémies ${ }^{20}$, le survivant des camps porte toujours en lui cette mort à laquelle il a échappé et parfois, se laisse rattraper par elle (Jean Améry, Tadeusz Borowski, Primo Levi, Piotr Rawicz).

La mort de l'autre, en camp, menace de disparition la communauté, ainsi que le montre Robert Antelme: «La mâchoire avide qui se décroche, le ventre vide qui s'affaisse, la mort du copain est une catastrophe. Mais la catastrophe, ce n'est pas seulement que ce copain soit mort. C'est que l'un de nous meure, que la mort arrive sur nous. » La catastrophe vient aussi de ce que cette mort ne constitue pas un événement. "Celui-là est mort. Ses amis s'en apercevront particulièrement, mais l'oublieront vite. Ça ne fait pas de bruit, rien ne s'arrête. Il meurt, c'est l'appel, il meurt, c'est la soupe, il meurt, on reçoit des coups, il meurt seul.»(Antelme, 1978, p. 103.)

L'opposition vivant/non vivant, qui est une des données fondamentales de la représentation du réel, est abolie dans les récits des camps. Le monde concentrationnaire a créé une figure inédite, celle du musulman dans les camps nazis, celle du dokhodiaga (crevard) dans les camps soviétiques. Pour celui qui a touché le fond, la frontière entre vie et mort devient perméable. "Je suis un crevard, un invalide patenté voué à l'hôpital, sauvé, arraché aux griffes de la mort par les médecins. Mais je ne vois aucun bien dans cette survie, ni pour moi ni pour l'État. [...] Être resté en vie est peut-être un bien, peut-être pas, c'est une question que je n'ai pas tranchée à ce jour. » (Chalamov, 2003, p. 1246.) Il existe dans ces camps toute une population qui se trouve dans un état intermédiaire entre la vie et la mort et quelques témoignages de cet état nous sont parvenus. En dépit de cette vérité que le Survivant n'est pas le vrai témoin, les témoignages littéraires sont précieux justement parce qu'ils tentent de saisir cet état particulier. Le survivant, dans nombre de cas, est celui qui a traversé la mort et qui en est revenu. Des études médicales sur les musulmans ont montré qu'il s'agit d'un état spécifique du corps que l'on ne saurait comparer à aucun autre. Le sociologue Wolfgang Sofsky, dans son analyse des camps de concentration nazis, le décrit de la façon suivante :

L'épuisement croissant abolissait la frontière entre la vie et la mort. Ce n'est pas un hasard si les musulmans rappelaient des «cadavres vivants». [...] Leur activité était descendue audessous du minimum animal. Ils ne voyaient et n'entendaient pratiquement plus rien, réagissaient seulement quand on leur hurlait dessus ou quand on les poussait pour qu'ils avancent. [...] La psychopathologie parle «d'anesthésie affective », d'une «annihilation », d'une «destruction radicale» du sens de la vie. Dans la casuistique clinique, le syndrome du musulman ne peut être confondu ni avec la maladie de la faim, ni avec les états de torpeur causés par la terreur, ceux que l'on rencontre souvent après les catastrophes naturelles.

(Sofsky, 1995, p. 250.)

Or, cet état entre la vie et la mort, certains témoins l'ont eux-mêmes connu, et leur témoignage y trouve à la fois sa légitimité et sa limite. Il s'agit d'un état qui échappe à la parole et qu'il faut pourtant revisiter, ce qui ne peut se faire qu'au prix de la perte de quelque chose d'essentiel, de ce vide existentiel, de cette absence à soi qui constitue le noyau de l'expérience concentrationnaire.

La destruction menée à son terme, l'œuvre accomplie, personne ne l'a racontée comme personne n'est jamais revenu pour raconter sa propre mort. Les engloutis, même s'ils avaient eu une plume et du papier, n'auraient pas témoigné, parce que leur mort avait commencé avant la mort corporelle. Des semaines et des mois avant de s'éteindre, ils avaient perdu la force d'observer, de se souvenir, de prendre la mesure des choses et de s'exprimer. Nous, nous parlons à leur place, par délégation (Levi, 1989, p. 82-83). Celui qui témoigne le fait à partir d'un corps reconstitué, il n'est donc plus installé dans la langue du musulman ou du dokhodiaga. Cette perte de la langue de l'expérience s'inscrit d'ailleurs dans la série des pertes que subit le prisonnier lors de son passage en camp et dont l'aboutissement est la mort. Elle se cristallise en une référence essentielle lors de la mise en parole de l'expérience: c'est autour d'elle que s'articule le projet d'œuvre littéraire appelée à dire le camp. Varlam Chalamov, qui a «touché le fond » à plusieurs reprises, consacre à cette question son récit «Le gant».

Atteint de pellagre, le détenu voit la peau de ses mains s'en aller intégralement, tel un gant. «Peut-on tenir une plume avec un gant pareil, qui devrait être conservé dans le formol ou l'alcool d'un musée, et qui repose dans la glace anonyme? [...] la peau qui a repoussé, cette peau neuve sur mes os, ont-ils vraiment le droit d'écrire? S'ils le font, que ce soit les mots qu'aurait 
pu tracer l'autre gant, celui de la Kolyma, le gant du forçat à la paume calleuse entamée jusqu'au sang par la rivelaine, aux doigts crispés sur le manche de la pelle. Seulement, cette main-là n'aurait pas écrit ce récit. Ces doigts-là sont incapables de se déplier pour prendre la plume et raconter leur histoire.»(Chalamov, 2003, p. 1246.)

On trouve la même image sous la plume de Charlotte Delbo:

Expliquer l'inexplicable. L'image du serpent qui laisse sa vieille peau pour en surgir, revêtu d'une peau fraîche et luisante, peut venir à l'esprit. J'ai quitté à Auschwitz une peau usée - elle sentait mauvais, cette peau - marquée de tous les coups qu'elle avait reçus, pour me retrouver habillée d'une belle peau propre, dans une mue moins rapide que celle du serpent, toutefois. Avec la vieille peau s'en allaient les traces visibles: les prunelles fixes au fond des orbites plombées, la démarche tirée en avant, les gestes peureux. Avec la nouvelle peau revenaient les gestes de la vie antérieure: se servir d'une brosse à dent, de papier hygiénique, d'un mouchoir, d'un couteau et d'une fourchette, manger posément, dire bonjour en entrant, fermer la porte, se tenir droit, parler, plus tard sourire des lèvres et, plus tard encore, sourire à la fois des lèvres et des yeux. [...] Il a fallu quelques années pour que la peau neuve se reconstitue, se consolide. Débarrassé de sa peau morte, le serpent n'a pas changé. Moi non plus, en apparence. [...] Comment se défaire de quelque chose enfoui beaucoup plus profond: la mémoire et la peau de la mémoire. Je ne m'en suis pas dépouillée. La peau de la mémoire s'est durcie, elle ne laisse rien filtrer de ce qu'elle retient, et elle échappe à mon contrôle. Je ne la sens plus. (Delbo, 1995, p. 12.)

La peau morte devient le symbole du texte initial perdu.

\section{LE TEXTE-SÉPULTURE}

L'absence de signes, l'idée de disparaître sans laisser de trace, constituent une source d'angoisse pour ceux des détenus à qui le camp laisse le temps de voir venir la mort. Voici ce que relate Gustav Herling dans Un Monde à part:

La mort dans les camps répand une autre forme d'effroi : son anonymat. Nous n'avions aucune idée de l'endroit où les morts étaient enterrés. [...] La certitude que personne n'apprendrait leur mort, que personne ne saurait jamais où ils avaient été enterrés, était l'un des plus grands tourments endu-

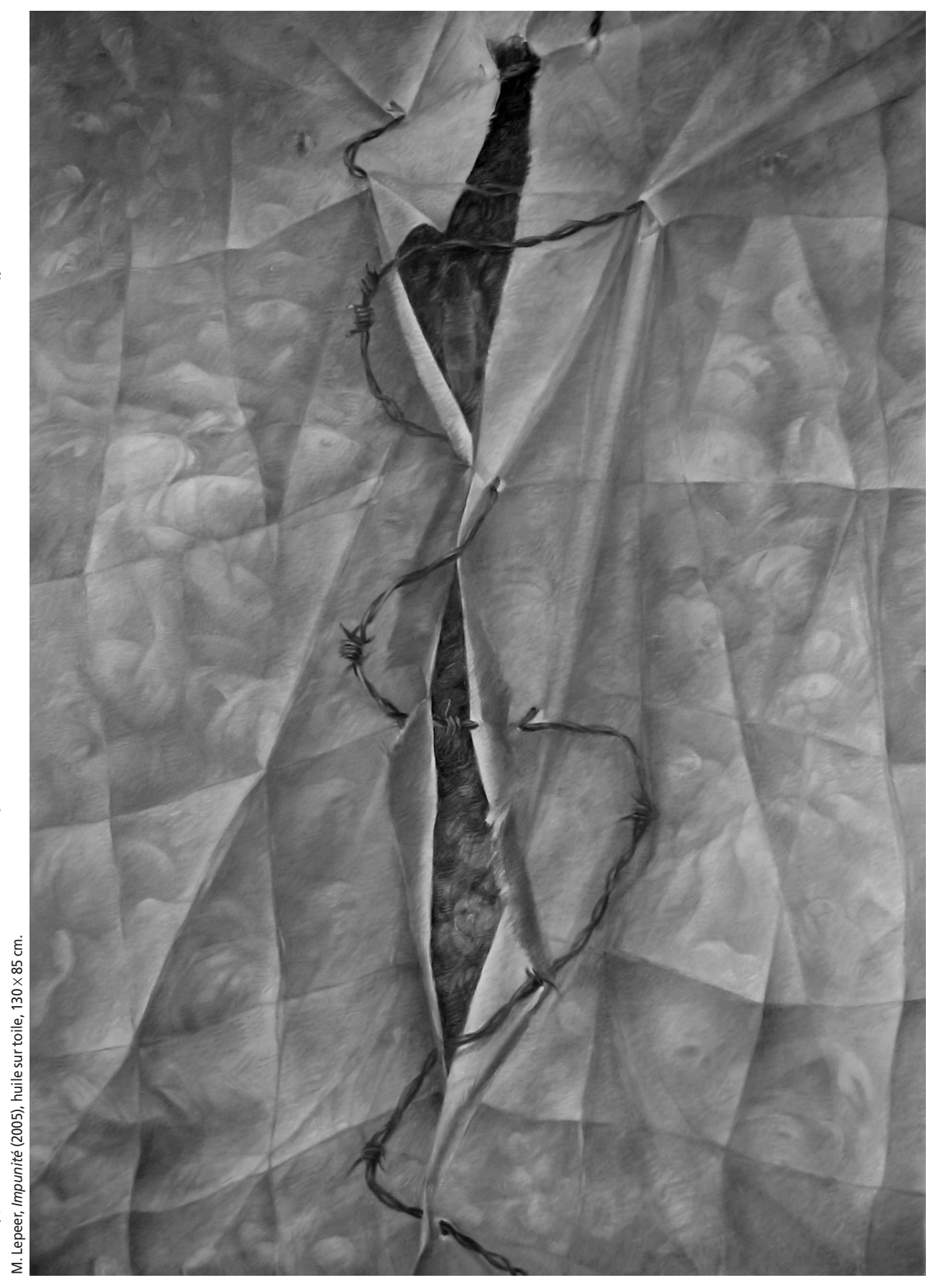

rés par les prisonniers. Il est possible d'être athée, de rejeter toute idée de survie après la mort; mais même dans ce cas, il est difficile d'accepter l'idée que la seule trace matérielle qui prolonge une vie humaine et lui donne une claire pérennité dans le souvenir des survivants, se trouvait ainsi effacée. Cet aspect de la peur de la mort, ou mieux, de l'annihilation complète, devint pour certains prisonniers l'objet d'une obsession. Il y avait de multiples accords secrets, conclus en quelque sorte bilatéralement, par lesquels les survivants s'engageaient à prévenir la famille du défunt de la date du décès et de l'emplacement approximatif de la tombe. (Herling-Grudzinski, 1995, p. 186-187).

Un de ces «accords secrets", cette fois au camp d'Auschwitz où règne la même obsession, fait l'objet d'un épisode de «Chez nous, à Auschwitz », récit de Tadeusz Borowski.

Un Prominent de mon bloc était malade; il avait mal, il avait de la fièvre et évoquait de plus en plus souvent la mort. Un jour, il m'a appelé. [...]

- Tu sais, je voudrais qu'on me mette à part. Pas avec les autres. Pas en tas avec eux. Tu comprends?

- N'aie pas peur, lui ai-je dit cordialement. Même, je te donnerai un drap. J'en toucherai un mot aux 
préposés aux cadavres. (Borowski, 1992, p. 165.)

On se rappelle le détour qu'a fait Ulysse pour enterrer un compagnon mort. Ne pas laisser le corps sans sépulture justifiait tous les risques. La paix du défunt en dépendait, ainsi que celle des vivants. On comprend donc aisément pourquoi dans les camps, la sépulture est chose impensable. L'entreprise d'anéantissement massif n'aurait pu être menée à terme si chaque mort recevait une sépulture. À l'inverse, une fois enclenché le processus de destruction, un tel rituel n'aurait plus aucun sens.

La société du camp n'avait plus qu'une existence révocable. L'agonie massive la séparait en une double masse: d'un côté la masse croissante des morts, de l'autre, la masse des survivants, d'où sortaient ceux qui s'ajouteraient à la montagne des morts. Ce n'était pas seulement que le régime punissait tous les rituels habituels du deuil. De telles pratiques n'auraient permis ni de comprendre ni de maîtriser la mort en masse. Une société menacée d'extermination ne pouvait plus se détacher des morts. Elle ne pouvait plus que tenter d'ignorer les mourants et de créer un mur social d'indifférence. (Sofsky, 1995, p. 256.)

Comme la disparition ou la destruction du cadavre représente la norme, un enterrement constitue à lui seul un événement. Il peut être l'objet d'un récit à part, comme c'est le cas dans «Tante Polia» de Chalamov. Tante Polia, une détenue qui faisait le ménage dans la maison du chef, était une privilégiée. Cela lui vaut d'être enterrée dans une vraie tombe, et, qui plus est, avec une croix. " On mit une croix, la première du cimetière. On pouvait la voir de loin. Bien qu'il n'y en eût qu'une, tout l'endroit prit l'aspect d'un vrai cimetière. Tous les malades qui pouvaient marcher allaient la voir. » (Chalamov, 2003, p. 153.)

Dans l'Espèce humaine de Robert Antelme, l'enterrement d'un camarade français, un des premiers morts au camp à une époque où il n'y avait pas encore beaucoup de décès, devient un événement emblématique, une stratégie face à l'engloutissement.

Trois hommes: deux pour porter le mort, la sentinelle. Un de plus, et ç'aurait été une cérémonie. Les SS ne l'auraient pas permis. Il ne faut pas que le mort puisse nous servir de signe. Il faut que nos morts disparaissent ici aussi, où il n'y a pas de crématoire. Notre mort naturelle est tolérée, comme le sommeil, comme de pisser, mais il ne faut pas qu'elle laisse de trace. Ni dans nos mémoires, ni dans notre espace. Il ne faut pas que le lieu

LES RITES D'ENTERREMENT PERMETTENT À LA COMMUNAUTÉ

DE PRÉSERVER L'ILLUSION DE SON IMMORTALITÉ.

où se trouve le mort puisse être situé.

(Antelme, 1978, p. 97-98.)

En fait, la sentinelle se détourne, permettant aux amis de faire une entaille sur un arbre, un repère.

Dans le camp de Birkenau, l'orchestre de femmes était dirigé par la violoniste Alma Rosé. Atteinte du cancer du cerveau, elle décéda au camp et fut enterrée dans un cercueil, signe de l'amour tout particulier que ses geôliers portaient à la musique. Lors du discours prononcé sur sa tombe, l'officier SS déclara que tout détenu modèle aurait le privilège d'être enterré ainsi.

L'absence de signes fait partie du projet d'anéantissement. Le seul signe possible, c'est le texte du témoin, qui se substitue à la sépulture. Celui qui prend la parole à la place des morts construit un tombeau. N'appelle-t-on pas ainsi une composition poétique ou musicale à la mémoire d'un défunt? Par exemple, Le Tombeau d'Edgar Poe de Mallarmé ou Tombeau pour Boris Davidovitch de Danilo Kis. Un grand nombre de textes sur les camps appartiennent à ce genre. Cette motivation est explicite, par exemple, dans «Oraison funèbre » de Varlam Chalamov. Mais même lorsqu'elle n'est pas inscrite dans le titre, elle reste essentielle. Qu'on se rappelle le préambule à l'Archipel du Goulag d'Alexandre Soljenitsyne : «Ceci est notre monument commun et amical à la mémoire de ceux qui ont été suppliciés et assassinés. » (Soljenitsyne 1974-1976, T. 1, p. 11). Les mots accomplissent l'œuvre de l'ensevelissement afin de rendre la survie aux vivants ${ }^{21}$, de séparer la communauté des vivants de celle des morts. Or l'œuvre n'est possible qu'à condition que les mots jaillissent du tombeau, à l'instant où le témoin lui-même, rendu à la vie, se sépare de son jumeau mort. Dans le récit «CherryBrandy», Varlam Chalamov imagine les derniers instants du poète Mandelstam, décédé dans une prison de transit de Magadan alors qu'il était en route vers les camps de la Kolyma. Décrite du point de vue d'un narrateur omniscient, cette agonie est narrée de l'intérieur, comme restituée en direct. Cette prison dont Chalamov fait le cadre de son récit est la fameuse maison Vaskov où il a séjourné lui-même en 1938, arrêté et condamné à mort alors qu'il se trouvait déjà en camp, puis sauvé in extremis, le fonctionnaire chargé de son affaire ayant été lui-même arrêté. Lieu de la mort provisoire, cette prison l'est aussi d'une prise de conscience radicale: les détenus qui viennent de quitter le monde libre et ne savent rien des camps ne peuvent pas communiquer avec ceux qui ont déjà traversé l'enfer. Le savoir d'un mort ne peut en rien profiter aux vivants ${ }^{22}$. L'homme chargé de ce savoir, celui qui a précédé Mandelstam dans la mort et qui en est revenu, apparaît dans le récit, un sourire énigmatique aux lèvres, comme un double du poète mourant. C'est seulement à ce prix que la parole se fait agissante. "Ce récit a été créé dès mon retour à la Kolyma, en 1954, à Rechetnikovo dans la région de Kalinine, où j'ai écrit nuit et jour pour fixer l'essentiel, laisser un témoignage, planter une croix sur une tombe, pour ne pas permettre que soit occulté un nom qui m'est cher à jamais, pour marquer cette mort qui ne saurait être ni pardonnée, ni oubliée. » Le récit n'aurait probablement pas vu le jour si l'auteur n'avait pas été convaincu de devoir créer une épitaphe : «À mon retour à Moscou, j'ai vu que dans chaque maison on trouvait des poèmes de Mandelstam. Cela s'est fait sans moi. Si je l'avais su, j'aurais peut-être écrit autre chose, différemment. » (Chalamov, 1998.)

Le texte-sépulture ne peut voir le jour que s'il est un texte-passage, lieu de la transmission de l'expérience des morts aux vivants, trace de cet instant insaisissable où le «je» du témoin se saisit du silence qui, dans l'autre, le disparu, fait écho à sa propre chute hors de lui-même. Il fait ainsi jaillir ce silence dans la parole, preuve de l'échange entre ceux qui sont revenus des camps et les autres, la majorité.

\section{Bibliographie}

ANTELME, R. (1978). L'Espèce humaine, Paris, Gallimard, coll. TEL.

APPLEBAUM, A. (2005). Goulag, Paris, Grasset.

ARENDT, H. (1972). Le système totalitaire, Paris, Le Seuil, coll. Points.

BENSOUSSAN, G., MESNARD, P. et SALETTI, C. (2005). Des voix sous la cendre, Manuscrits des Sonderkommandos d'Auschwitz-Birkenau, Paris, Calmann-Lévy.

BOROWSKI, T. (1992). Le Monde de pierre, Paris, Christian Bourgois.

CANETTI, E. (1986). Masse et puissance, Paris, Gallimard, coll. TEL.

CHALAMOV, V. (2003). Récits de la Kolyma, Lagrasse, Verdier.

CHALAMOV, V. (1998). Sobranie socinenij $v$ 4eh tomah, t. 4, p. 364.

DELBO, C. (1995). La Mémoire et les jours, Paris, Berg international. 
FREUD, S. (1988). «Actuelles sur la guerre et la mort», OCF-P, t. XIII, Paris, PUF.

FROMER, R. (1993). The Holocaust Odyssey of Daniel Bennahmias, Sonderkommando, Tuscaloosa, University of Alabama Press.

GROSSMAN, V. (1993). «L'enfer de Treblinka » in Années de guerre, Paris, Autrement.

HERLING-GRUDZINSKI, G. (1993). Un monde à part, Paris, Gallimard.

HILBERG, R. (1988). La Destruction des Juifs d'Europe, Paris, Fayard.

HÖSS, R. (1995). Le Commandant d'Auschwitz parle, Paris, La Découverte.

LANZMANN, C. (1985). Shoah, Paris, Fayard.

LEVI, P. (1989). Les Naufragés et les rescapés, Paris, Gallimard, coll. Arcades.

MÜLLER, F. (1980). Trois ans dans une chambre à gaz d'Auschwitz, Paris, Pygmalion.

RIQUEUR, P. (2000). La Mémoire, l'histoire, l'oubli, Paris, Le Seuil.

SOFSKY, W. (1995). L'Organisation de la terreur, les camps de concentration, Paris, Calmann-Lévy.

SOLJENITSYNE, A. (1974-1976). L'Archipel du Goulag, Paris, Le Seuil.

SOLJENITSYNE, A. (1976). Une journée d'Ivan Denissovitch, Paris, Julliard.

WIEVIORKA, A. (1992) Déportation et Génocide, Paris, Plon.

ZMIJEWSKAZ-WISNIEWSKA, A. (1965). «Zeznania szefa Krematorium Ericha Mushsfeldta na temat bylego obozu konzentracyjnego w Lublinie (Majdanek)», in Zeszyty Majdanka, I.

\section{Notes}

1. Je rappelle ici la phrase de Primo Levi, devenue emblématique de la difficulté à témoigner: «Nous, les survivants, ne sommes pas les vrais témoins. [...] Nous, les survivants, nous sommes une minorité non seulement exiguë, mais anormale: nous sommes ceux qui, grâce à la prévarication, l'habileté ou la chance, n'ont pas touché le fond. Ceux qui l'ont fait, qui ont vu la Gorgone, ne sont pas revenus, ou sont revenus muets, mais ce sont eux, les "musulmans", les engloutis, les témoins intégraux, ceux dont la déposition aurait eu une signification générale. » (Levi, 1989, p. 82.) Presque tous les auteurs qui ont parlé des camps le disent d'une façon ou d'une autre.

2. Toutefois, à partir des années 1930, il apparaît dans le discours officiel que seuls les criminels de droit commun sont rééducables, les politiques ne le sont pas. Par "politiques », il faut cependant entendre les détenus condamnés au titre de l'article 58, en fait, les victimes choisies arbitrairement selon la purge du moment. Il y avait très peu de vrais opposants dans les camps soviétiques.

3. Lors de leur procès, des nazis prétendirent que le but essentiel des camps était l'industrie d'armement et que les autres objectifs, y compris l'extermination, étaient soumis à cet objectif principal (Rudolf Höss, par exemple).

4. À Auschwitz, ce fut la maison J.A. Topf und Söhne d'Erfurt qui emporta le marché et livra quatre chambres à gaz au printemps 1943.

5. Parmi les raisons invoquées par les responsables des camps lors de leur procès, il y avait celle que les corps en décomposition risquaient de contaminer l'eau potable sous l'effet de la chaleur (été 1942). Cette justification montre combien les Juifs étaient associés pour les nazis à la contagion. On trouve cette crainte de la maladie dans de nombreuses dépositions, ainsi que dans le témoignage du docteur Franz Grassler interrogé par Claude Lanzmann (Lanzmann, 1985, p. 221-239).

6. En réalité, ce principe ne s'appliquait pas toujours, puisque les familles des ennemis du peuple étaient également déportées. Par ailleurs, la déportation des peuples se faisait sur un critère ethnique et non plus politique. Toutefois, la faute invoquée était toujours rattachée à une trahison, à un point de rupture. Ainsi, les victimes de la persécution antisémite étaient officiellement coupables du crime du cosmopolitisme, et non d'une faute congénitale.

7. Par exemple, le colonel Garanine.

8. Pour les témoignages écrits, voir notamment Müller (1980) ainsi que Bensoussan et al. (2005). Pour les témoignages oraux, voir Lanzmann (1985) ainsi que Fromer (1993).

9. Notamment, la déposition du commandant du crématoire de Lublin-Majdanek, Erich Mushsfeldtdans Zmijewskaz-Wisniewska (1965) et celle de Rudolf Höss, dans Höss (1995).

10. Ces témoins-là n'étaient pas toujours en mesure d'analyser ce qui s'offrait à leurs yeux. On doit une reconstitution fidèle du processus de la mise à mort à la plume de Vassili Grossman «L'enfer de Treblinka » dans Grossman (1993).

11. Sur toutes les étapes de la concentration et de l'extermination, voir Hilberg (1988).

12. Voir également, sur cette question, le chapitre «Les mourants» dans Applebaum (2005).

13. Les centres de mise à mort et les principaux camps de concentration sont répertoriés notamment par Annette Wieviorka dans Wieviorka (1992).

14. Exception faite des Allemands condamnés pour des crimes de droit commun et qui jouissaient d'importants privilèges par rapport aux autres détenus.

15. Voir aussi, dans Une journée d'Ivan Denissovitch: «Ferme la porte, fumier! [...] Sale cochon! [...] Ces ordures, ça ne sait rien faire [...]. De la merde! Voilà ce qu'on devrait leur filer. » (Soljenitsyne, 1976, p. 28.)

16. Un récit de Varlam Chalamov, «Le graphite ", est consacré à ce sujet: ce numéro, écrit au crayon de papier, resterait gravé pour l'éternité, résistant au gel et aux intempéries, constituant ainsi une trace indélébile. En vérité, le bois ou le contreplaqué qui servait à confectionner ces plaques se décomposait souvent, rendant impossible l'identification (Chalamov, 2003, p. 996-1001).
17. Hannah Arendt définit l'une des finalités de l'État totalitaire comme la "production » d'un certain type d'humain, dépourvu de toutes les caractéristiques de l'espèce à l'exception des fonctions de reproduction. On pourrait voir justement dans le cadavre la phase extrême de ce processus.

18. Pourtant, les desseins des bourreaux sont parfois déjoués par la matière elle-même, ainsi que le relate Chalamov dans son récit «Prêt-bail»: «La fosse, la tombe commune des détenus - une grande fosse en pierre bourrée jusqu'en surface de cadavres non décomposés - avait commencé à s'ébouler dès 1938. Les corps s'étaient mis à glisser sur le flanc de la montagne, révélant le secret de la Kolyma. À la Kolyma, on dépose les corps non pas dans la terre, mais dans le rocher. La pierre garde et révèle les secrets. [...] Tous nos proches qui ont péri à la Kolyma, tous ceux qui ont été fusillés, battus à mort, saignés à blanc par la faim, tous peuvent être identifiés même après une dizaine d'années. Il n'y avait pas de fours crématoires à la Kolyma. Et les cadavres attendaient dans le roc, dans le permafrost. »(Chalamov, 2003, p. 513.)

19. Voir le témoignage de Motke Zaïdl et de Itzhak Dugin in Lanzmann, 1985, p. 27-28.

20. Sur ce sujet, voir Freud (1988).

21. «C'est en tout cas la fonction du discours comme lieu de la parole d'offrir aux morts du passé une terre et un tombeau ». (Riqueur, 2000.)

22. «Personne ne lui posa de questions bien qu'il y eût très peu de gens venus de la taïga dans ce camp de transit et que tous les autres fussent destinés à y aller. Et ils le comprenaient parfaitement. Et c'est justement pour cela qu'ils ne voulaient rien savoir de l'inéluctable taïga. Et c'était aussi bien, d'après Andreïev. Ils n'avaient pas besoin de savoir tout ce que lui avait vu. On ne pouvait rien éviter: ici, les supputations étaient inutiles. À quoi bon une angoisse supplémentaire? Ceux qui se trouvaient ici étaient encore des hommes. Andreïev, lui, représentait des morts. Et son savoir, celui d'un homme mort, ne pouvait leur être d'aucune utilité, à eux qui étaient encore vivants. »(Chalamov, 2003, p. 251.) 